\title{
THE RESULTS OF THE RECONSTRUCTION OF THE COEFFICIENTS OF THE NUTATION FOR THE RIGID EARTH MODEL AND THEIR COMPARISON WITH NUMERICAL INTEGRATION
}

\author{
J. SOUCHAY and H. KINOSHITA \\ Tokyo National Astronomical Observatory, Mitaka Shi \\ Tokyo 181, JAPAN
}

\begin{abstract}
In view of the present accuracy of the astrometric observations and of the development of the theory of the nutation, it became necessary to make a complete revision of this theory for a rigid Earth model. We present the results of our recent one (Kinoshita and Souchay, 1989), which includes planetary effects and second order effects no considered in the previous tables (Kinoshita, 1977). We analyze the difference between these tables and the new ones providing from the revision above and the comparison between the theory and numerical integrations recently performed (Kubo and Fukushima, 1987; Shastok et al., 1987; Shastok et al., 1989). The results of this comparison are much better after revision than before.
\end{abstract}

\section{Introduction}

A complete reconstruction of the theory of the nutation for the rigid Earth model was recently performed by Kinoshita and Souchay (1989). At first they calculated the terms due to the first-order potential of the Moon and of the Sun with a level of truncation of 0.01 milliarcsecond instead of 0.1 milliarcsecond (Kinoshita, 1977), and by using up-todate semi-analytical theories VSOP82 (Bretagnon, 1982) and ELP2000 (Chapront-Touzé and Chapront, 1983). Moreover, they accounted for scond-order terms due to smaller components of the gravitational field of the Earth, and to a coupling effect between the orbital motion of the Moon and the rotational motion of the Earth. At last, they included planetary influences which can be selected into three categories: their direct torques on the Earth, their perturbation on the orbital motion of the Moon, and their perturbation on the orbital motion of the Earth. In the following we summarize the consequences of all these contributions.

\section{Comparison between Old and New Tables}

The Hamiltonian associated with the motion of rotation of the Earth can be divided in two parts according to their nature. The first-order one is the part (not combined) directly coming from the main component of the disturbing potential, that is saying the component containing the zonal harmonic $J_{2}$ of the Earth. Thus, rigorously speaking, we can include in this part the direct and the indirect effects of the planets. In his old tables Kinoshita (1977) included only the terms due to the lunisolar potential. He found 106 coefficients 
in longitude, and 54 in obliquity, up to 0.1 mas (notice that because of the truncation, Kinoshita kept in fact any term bigger than 0.05 mas). Within the same range, we find two supplementary terms in longitude (and the two corresponding terms in obliquity), which are written, in mas:

$$
\Delta \psi=0.13 \sin (2 l-2 F+2 D-\Omega)+0.12 \sin \left(-l+l^{\prime}+D+\Omega\right)
$$

As far as the lunisolar effect only is concerned, 148 terms in longitude and 95 terms in obliquity must be added only by accounting for all the coefficients up to 0.01 mas instead of 0.1 mas. Notice also that the total planetary effect is notable, with 180 terms at all, and some of them bigger than 0.1 mas.

Now, if we consider the revision of the theory at the second order in Kinoshita and Souchay (1989), we observe that it produces important changes in the low frequencies, when inserting a contribution due to the dynamical interaction between the figure of the Earth and the orbital motion of the Moon (Kubo, 1982; Kinoshita, 1988). Besides this main effect, other subsequent terms are providing from the influence of tesseral $\left(C_{22}\right.$ and $\left.S_{22}\right)$ and zonal $\left(J_{3}\right)$ harmonic coefficients of the gravitational field of the Earth on the expression of the lunisolar potential.

In table 1 , we indicate the number of new coefficients involved in the theory both at the first order and at the second order, according to their origin. The most significant corrections after reconstruction concern the terms of argument $\Omega$ and $2 \Omega$, principally because of the dynamical interaction described above. They are written:

$$
\begin{gathered}
\delta(\Delta \psi)=-0 ! .00026 \sin \Omega+0 ! .000117 \sin 2 \Omega \\
\delta(\Delta \varepsilon)=0 \prime .00095 \cos \Omega-0.00022 \cos 2 \Omega
\end{gathered}
$$

\section{Comparison with Numerical Integration}

To compute the nutation by means of numerical integration is a very useful task in order to check the results given by the theory. This has already been done by Kubo and Fukushima (1987) and Schastok et al. (1987) before the revision of the theory (Kinoshita and Souchay, 1989), and again by Schastok et al. (1989) after this revision. All the authors used Woolard's theory as a basis. The two former works look very much in agreement one to each other, showing relatively big discrepancies with the values given by Kinoshita's tables (1977). Besides, Kubo and Fukushima give explicitely the following differences for the terms of argument $\Omega$ and $2 \Omega$ :

$$
\begin{gathered}
\delta(\Delta \psi)=0^{\prime \prime} .0006 \sin \left(\Omega-26^{\circ}+0^{\prime \prime} .0013 \sin \left(2 \Omega-2^{\circ}\right)\right. \\
\delta(\Delta \varepsilon)=0 \prime \prime 0008 \cos \left(\Omega+26^{\circ}\right)-0^{\prime \prime} .0003 \cos \left(2 \Omega+37^{\circ}\right)
\end{gathered}
$$

which are very similar to the corresponding analytical corrections (2). Furthermore, in the curves given by Schastok et al. (1987) as well as in the power spectra given by Kubo and Fukushima (1987) semi-annual discrepancies appear clearly whose the amplitudes (about 0.1 mas in longitude, 0.05 mas in obliquity) correspond exactly to the amplitudes of the quasi semi-annual corrections between Kinoshita (1977) and Kinoshita and Souchay (1989). At 
last, when comparing their results of numerical integration with the new series by Kinoshita and Souchay (1989), Schastok et al. (1989) show that the discrepancy is no more than 0.3 mas in longitude and 0.2 mas in obliquity, after fitting some of the biggest coefficients. The same comparisons with the old series of Kinoshita (1977) gives discrepancies of the order of 1.5 mas and 1. mas respectively. This big improvement is partly due to the introduction of the planetary terms in the new series. It is also worthy to remark that the big discrepancy for the term of argument $2 \Omega$ noticed by Kubo and Fukushima (equations (3)) disappears completely after the analytical corrections (2), both in longitude and in obliquity.

Notice that we will soon achieve ourselves a complete determination of the nutation for the rigid Earth model by numerical integration, starting from Kinoshita's basic equations (Kinoshita, 1977). Preliminary comparisons with the tables given by Kinoshita and Souchay (1989) within a few years and without any fit, show very small dicrepancies, with a mean square error lower than 0.05 mas for $\Delta \psi$ and $\Delta \varepsilon$.

\begin{tabular}{|c|c|c|c|c|}
\hline \multirow[t]{2}{*}{ Part } & \multicolumn{2}{|c|}{ Longitude } & \multicolumn{2}{|c|}{ Obliquity } \\
\hline & $\begin{array}{c}\text { Number of new } \\
\text { terms } \\
\geq 0.01 \text { mas }\end{array}$ & $\begin{array}{c}\text { Sum } \\
\text { (in phase) }\end{array}$ & $\begin{array}{c}\text { Number of new } \\
\text { terms } \\
\geq 0.01 \text { mas }\end{array}$ & $\begin{array}{c}\text { Sum } \\
\text { (in phase) }\end{array}$ \\
\hline First order & & & & \\
\hline Moon (main problem) & 145 & $2.5 \mathrm{mas}$ & 93 & $1.6 \mathrm{mas}$ \\
\hline Sun (keplerian motion) & 3 & $0.1 \mathrm{mas}$ & 2 & 0.1 mas \\
\hline Planets (indirect effect) & 93 & $1.6 \mathrm{mas}$ & 37 & 0.4 mas \\
\hline Planets (direct effect) & 36 & $0.8 \mathrm{mas}$ & 14 & $0.2 \mathrm{mas}$ \\
\hline Second order & & & & \\
\hline Triaxiality & 7 & $0.1 \mathrm{mas}$ & 3 & 0.05 mas \\
\hline & 6 & $0.2 \mathrm{mas}$ & 3 & 0.1 mas \\
\hline Coupling effect & 7 & $1.9 \mathrm{mas}$ & 7 & 0.5 mas \\
\hline Total & 297 & $7.2 \mathrm{mas}$ & 159 & $3.0 \mathrm{mas}$ \\
\hline
\end{tabular}

Table 1. Number of new terms included in the new tables of the nutation (Kinoshita and Souchay, 1989) and their sum, in phase. 


\section{References}

Bretagnon P. (1982), Astron. Astrophys. 114, 278-288

Chapront-Touzé M., Chapront J. (1983), Astron. Astrophys., 124, 50-62

Kinoshita H. (1977), Celest. Mech. 15, 277-326.

Kinoshita H. (1988), BIH : Annual Report for Year 1987, D-103.

Kinoshita H., and Souchay J. (1989), "Theory of the Nutation at the Second Order", Celest. Mech., in press.

Kubo Y. (1982), Celest. Mech. 26, 96-112.

Kubo Y., and Fukushima T. (1987), in: G. Wilkins and A. Babcock (Eds), The Earth's Rotation and Reference Frames for Geodesy and Geodynamics, Proc. of IAU Symposium. No. 182, Reidel.

Schastok J., Soffel M., Ruder H. (1987), "Variations in Earth Rotation", Proc. IUGG Symposium, U4

Schastok J., Soffel M., Ruder H. (1989), "Numerical Derivation of Forced Nutation Terms for a Rigid Earth," Comm., in press.

\section{Discussion}

HugHes: Can you state which of the three new effects- $\mathrm{J}_{3}$, indirect perturbations, and direct perturbations - has contributed most to the improvement, or are they more-or-less equal?

Souchay: The effect of $\mathrm{J}_{3}$ is small compared with the two others $(\approx 0.1 \mathrm{mas})$. The indirect and direct effects are of the same order, but the indirect one contributes the most.

BARKIN: The unperturbed rotational motion of the Earth in your paper is the Eulerian motion of a rigid body. How does the difference between the Euler period and the Chandler period in the pole motion influence the perturbed rotation of the Earth, which is constructed in your paper in analytical form?

Souchay: Since the change of period between the Euler period and the Chandler period characterizes the nature of the model of the Earth which is chosen, it would be nonsense to study the difference mentioned because we only deal with the rigid-Earth model. Anyway, it seems that this difference should not modify the equations for the perturbed motion.

YATSKIv: (1) Zhu and Groten have pointed out some differences between your calculations of nutation and their work. What are the causes? (2) Could you explain the differences between the new analytical nutation and numerical integrations?

SouchaY: (1) Zhu and Groten did not take into account some terms. (2) It is due to the truncation in the series, or to the combination of terms of very small amplitude $(\approx 0.01$ mas $)$ in the second-order theory. 